\title{
Investigating the feasibility of a wireless motion capture system to aid in the rehabilitation of total knee replacement patients
}

\author{
Mobolaji Ayoade \\ Multimodal Research Group \\ School of Engineering \& Computing \\ Glasgow Caledonian University \\ Glasgow, UK \\ mobolaji.ayoade@gcu.ac.uk
}

\author{
Lee Morton \\ Multimodal Research Group \\ School of Engineering \& Computing \\ Glasgow Caledonian University \\ Glasgow, UK \\ lee.morton@gcu.ac.uk
}

\author{
Lynne Baillie \\ Multimodal Research Group \\ School of Engineering \&Computing \\ Glasgow Caledonian University \\ Glasgow, UK \\ 1.baillie@gcu.ac.uk
}

\begin{abstract}
Currently, patients are discharged to their homes after successful total knee replacement (TKR) surgery with a standard exercise booklet that contains instructions on how to carry out home exercises. This paper investigates the feasibility of building a low cost inertial motion capture system to provide patients with real time visualization of biomechanical data while performing home based rehabilitation exercises. Commercially available motion capture systems suitable for clinical diagnosis or rehabilitation, such as Vicon are expensive, require professionals to setup and have complex calibration procedures. The accuracy of our proposed motion capture system is assessed using a Vicon optical motion tracking system. The measurements are compared using a simple total angular displacement analysis.
\end{abstract}

Keywords- knee; exercises; rehabilitation; home; motion capture; visualization;

\section{INTRODUCTION}

After total knee replacement surgery, the patient is usually monitored in the hospital ward and closely supervised by a physiotherapist in the inpatient rehabilitation unit. During this period, the patient is also taught how to carry out home rehabilitation exercises. The patient is then discharged and referred to outpatient therapy when deemed fit to go home. Outpatient therapy can be home based, in which the patient carries out individual exercises alone, one-to-one with a physiotherapist or based in groups in a rehabilitation centre.

The goal of all rehabilitation programs is to restore normal motor function to the patient. Inpatient rehabilitation has the advantage that the patient has access to monitoring equipment, close supervision and guidance from a physiotherapist and/or a nurse who ensure that the rehabilitation exercises are targeted and carried out correctly [2]. However, this approach is costly and increases demands on hospital resources [3, 4]. Moreover, giving the need to reduce cost of health care service delivery and the increasing number of people with joint problems, more and more interest has been drawn toward the development of effective home based rehabilitation schemes [4].

Home based rehabilitation reduces the need for face-to-face interaction between physiotherapists and patients [4]. Unfortunately, its effectiveness is largely dependent on a

Sponsored by Life Long Health and Wellbeing (LLHW) patient's self-motivation to correctly carry out an exercise program with very little or no support or feedback [2]. Previous studies have shown that provided the rehabilitation given is of sufficient high quality, outpatient home rehabilitation has similar outcomes with inpatient rehabilitation [2]. The quality of home or community rehabilitation can thus be improved by incorporating the strength of other therapies [1, 2] i.e. appropriate feedback.

It is hoped that by providing a system that can provide biomechanical feedback to a patient at home, motivation will be maintained, exercises will be carried out correctly and recovery will be enhanced. some of the strengths of inpatient therapy can be brought to patients in home or community based rehabilitation settings.

\section{RELATED WORK}

Many researchers have studied the use of technology to enhance home based rehabilitation of patients [4-6]. Although, the majority of research has been in laboratories or controlled environments and as such little evidence exists to show how these systems function in natural home settings. These studies address the application of position sensors, such as goniometry, pressure sensors and switches, magnetometers, and inertial sensors to rehabilitation [4].

Inertial sensors (i.e. accelerometer and gyroscopes) and magnetometers have been widely used for human motion tracking [7-9]. A real-time human arm motion detector to aid home-based rehabilitation of stroke patients consisting of two tri-axial inertial sensors was shown to be comparable to commercial optical motion capture systems [10-12]. A pilot study trial for motor rehabilitation of stroke patients has also been carried out using miniature wireless inertial sensors [13, 14]. In another study, wireless inertial measurement units combined with pressure sensor systems were used to capture 3D knee joint angle motion [15].

Robot-aided systems typically consist of mechanical structures and sensor technologies such as inertial sensors for tracking human motion. For instance, a robotic exercise aid has been designed to guide and control physical therapy as well as record motion [16]. Other researchers have used robotics for 
upper limb rehabilitation of stroke patients [5, 6, 17-20]. Unfortunately, there is little research on the application of robotics to knee rehabilitation.

Optical motion capture has found widespread use in human motion tracking and is very accurate in position estimation [4]. Cameras either track the position of markers attached to the subject (marker based) or use image processing techniques (marker-less based). Gait clinics often use optical based systems to conduct gait analyses to diagnose gait related diseases and monitor effectiveness of treatment procedures [22]. Optical motion capture systems are not suitable for home rehabilitation because they are very expensive and usually require a dedicated technician to operate [4]. For example, the Vicon system can cost up to $€ 200,000$.

\section{RESEARCH AIMS AND OBJECTIVES}

The aim of our research is to produce a motion capture system that is suitable for home or community based rehabilitation applications, initially focusing on total knee replacement patients. The motion capture system must be wireless so as to allow for unrestricted movement of the patient. It should also have low weight, miniature form-factor and low-power consumption.

The first stage in producing such a rehabilitation system is to validate a pre-built inertial and magnetic sensor board as a possible platform for a motion capture system. An accuracy of $\pm 3^{\circ}$ is sufficient for motor rehabilitation as this is better than an average physiotherapist can discern by eye [12].

\section{System ARCHITECTURE AND DESIGN}

Our inertial motion capture systems consist of 3-axis magnetic, angular rate and gravitational (MARG) sensor arrays. One sensor is attached to each major limb segment and measures the 3D orientation of that segment. These sensors are connected wirelessly to a coordinator node connected to a PC [Fig 1]. The orientation data, when combined with a skeletal model, can be used to reconstruct a subject's posture. The system uses gyroscopes to measure the rotational movement of limb segments. The accelerometers and magnetometers are used to correct any drift caused by gyroscope measurement errors. Some other systems operate without the use of magnetic sensors to correct gyroscope drift [23].

Typically one of two types of software orientation filter are used, complimentary or Kalman. Complimentary filters produce orientation estimates by integrating the angular rate measurements and then correcting the drift caused by measurement errors using the gravitational and magnetic field measurements [23]. Kalman filters are usually more complex and often involve iterative processes [9] so are therefore more computationally expensive than a complimentary filter. They do however have the ability to deal more effectively with errors [24].

The pre built sensor boards being validated in this paper are available from Sparkfun Electronics [25]. These include three axis accelerometers, gyroscopes and magnetometers. An ATMEGA328P microcontroller is included on these boards and we have tested its suitability for processing orientation

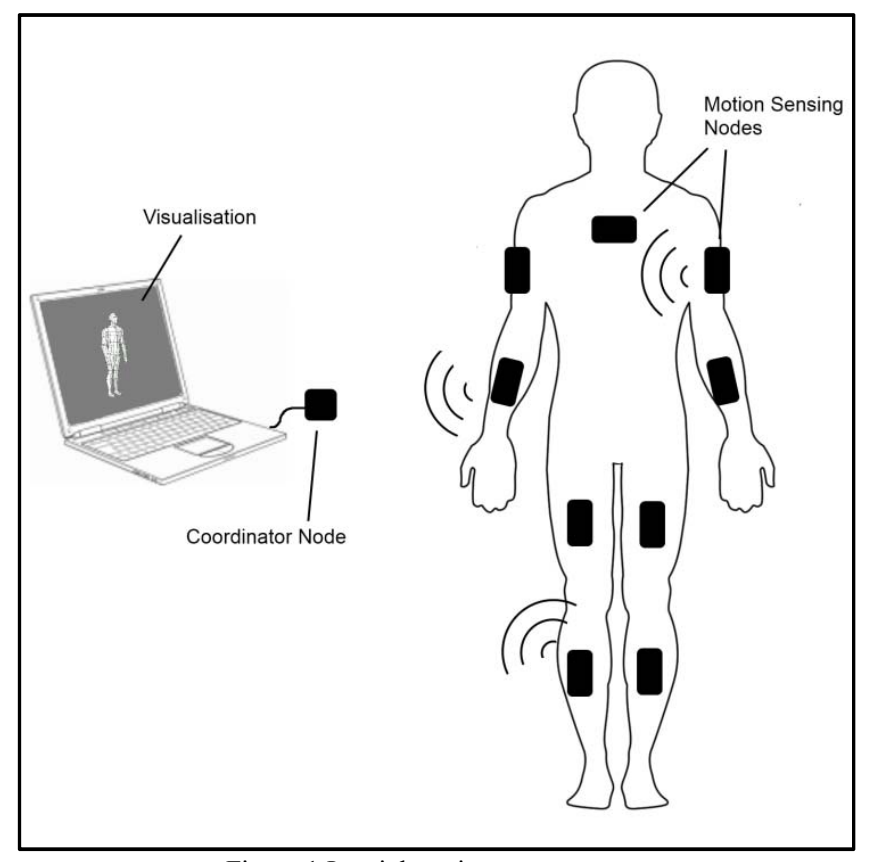

Figure 1 Inertial motion capture system

calculations on board. These boards also have a UART (serial) output for connection to various radio technologies.

\section{EXPERIMENTAL SETUP}

The sensor board is connected to a host PC via a wireless $\mathrm{XBee}$ radio network. Orientation calculations can either be performed on board the sensor or on the host PC depending on desired setup. For the purposes of this experiment the calculations were performed in real time onboard the PC. A complimentary filter was used for ease of implementation.

To validate the potential accuracy of our sensors, a Vicon optical 3D motion analysis system was used to measure the orientation of the sensor under dynamic movement. Vicon can produce accurate position information (the calibration residuals were $<0.5 \mathrm{~mm}$ on the day we tested) [4]. The inertial sensor was attached to a rigid wooden board with three optical markers attached at three edges of the board. The motion capture system tracks the position of these markers in $3 \mathrm{D}$ space and can calculate the orientation of the sensor.

In this experiment the two data streams were captured on separate PCs. This means that the recorded results will inevitably be offset from each other slightly in time. In order to synchronize these data streams during post experiment analysis a distinctive movement pattern was performed at the beginning of each capture session. This movement pattern was used to determine a time offset value between the two data streams by selecting a point corresponding to the same feature from each set of data. The time difference between these two points is the time offset value. In practice we found that the single offset value was not sufficient to align the data from the two streams as the calculated sampling rates were slightly different. Fortunately, there was enough detail to select another pair of points, towards the end of the captured data, to synchronize the data streams more accurately. 
The reference frame of the optical motion capture system is different from the one used by the sensor for several reasons. The optical system determines the vertical axis from placing a calibration wand on the floor, the inertial motion sensors take the vertical direction by measuring gravity directly. The optical motion capture system defines the horizontal axes to be in line with the walls of the room, the inertial system defines them using the earth's magnetic field. In addition to this, any misalignment caused by mounting the sensors to the wooden board will contribute to the offset in reference frames. To compare the two sets of data with $3 \mathrm{D}$ orientation information one reference frame would have to be transformed into another. We have opted for a simpler approach of defining a start orientation and measuring a scalar angular displacement from that orientation. The sensor was left stationary for several seconds before each capture session. This stationary position was taken to be zero angular displacement as a reference for each data set.

Each movement sequence performed involved rotating the inertial sensor about each axis approximately $90^{\circ}$ in one direction, $180^{\circ}$ in the opposite direction then $90^{\circ}$ to return it to its initial position. This can be seen in Fig 2 as a double hump. Because our measurement of angular displacement is always a positive scalar, the direction of movement is not obvious. The second hump is movement in the opposite direction of the first. The peak of the first hump corresponds to the end of the first $90^{\circ}$ movement, the bottom of the valley in-between the peaks corresponds to the midway point of the $180^{\circ}$ movement in the opposite direction (when the sensor is very close to its starting position). The next peak corresponds to the end of this movement when the sensor has moved a further $90^{\circ}$ away. This sequence was repeated for each axis of each of the sensors tested.

\section{RESULTS}

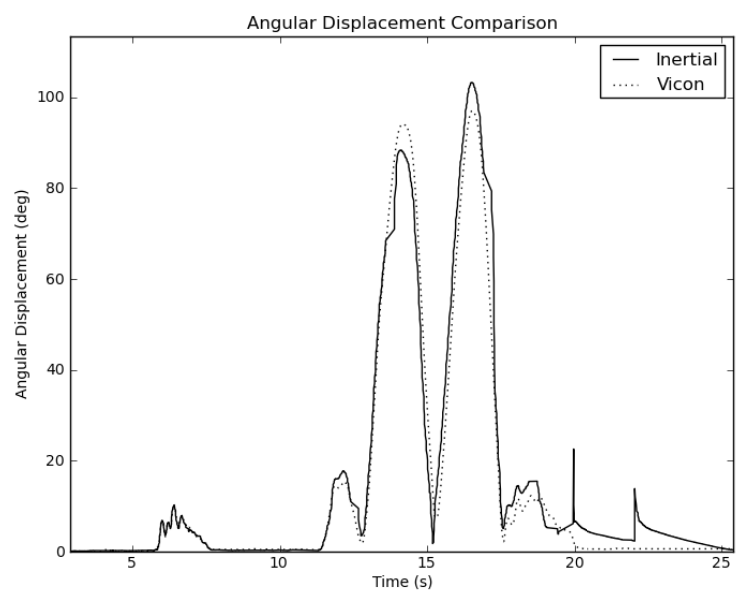

Figure 2 Angular displacement of sensor 3, z-axis, compared to the optical motion capture system. The two large peaks in the center are produced from a motion approximately $90^{\circ}$ in one direction, $180^{\circ}$ in the other then $90^{\circ}$ back to the starting position.

The orientation filter has a drift correction mechanism. Upon start-up this drift correction requires a significant amount of time to converge on a stable value. If the drift correction has not converged by the time the experiment has started then the drift correction process will continue and produce a significant amount of error. During each of our test capture sessions the drift correction algorithm was allowed two minutes to converge. Upon analysis, this time was in fact too short and significant drift artifacts could be seen in the results. Results with obvious drift artifacts were discarded as we don't believe they will be representative of a fully functioning system. There were sufficient results to be able to make an approximate estimate of the system's accuracy.

Drift correction is a part of all our result sets and can be seen in Fig 2 towards the end, after the 20s mark. In this case there was about 5 seconds of drift correction after the movement sequence for it to converge to the correct value.

TABLE I. DIFFERENCES IN MEASURED ANGULAR DISPLACEMENT

\begin{tabular}{|c|c|c|c|c|}
\hline \multicolumn{2}{|c|}{} & \multicolumn{3}{|c|}{ Angular Displacement (degrees) } \\
\cline { 3 - 5 } \multicolumn{2}{|c|}{} & Vicon & Inertial & Error \\
\hline \multirow{2}{*}{$\begin{array}{c}\text { Sensor 1 } \\
\text { y-axis }\end{array}$} & Peak 1 & 82.0 & 88.9 & 6.9 \\
\cline { 2 - 5 } & Peak 2 & 79.6 & 83.5 & 3.9 \\
\hline $\begin{array}{c}\text { Sensor 2 } \\
\text { y-axis }\end{array}$ & Peak 1 & 83.6 & 87.6 & 4.0 \\
\cline { 2 - 5 } & Peak 2 & 80.5 & 83.1 & 2.6 \\
\hline $\begin{array}{c}\text { Sensor 3 } \\
\text { z-axis }\end{array}$ & Peak 1 & 94.1 & 88.3 & 5.8 \\
\cline { 2 - 5 } & Peak 2 & 97.1 & 103.3 & 6.2 \\
\hline \multicolumn{2}{|c|}{} & & $\mathbf{4 . 9} \pm \mathbf{1 . 5}$ \\
\hline
\end{tabular}

The difference in angular displacement peak heights of each set of valid data was compared with the angular displacement measured by the Vicon system [Table1]. The measurement error is taken to be the difference between the heights of two corresponding peaks. Measurements for both peaks are shown in table 1. Although we took measurements for each of the 3 axes of the 4 sensors tested, only the $y$-axis in sensor 1 and 2, and z-axis of sensor 3 produced meaningful results. Other data had to be discarded for reasons described above.

\section{CONCLUSION AND FUTURE WORK}

The measurement error of the sensors was found to be about $5^{\circ}$. This is not within our target $\pm 3^{\circ}$ that a physiotherapist is able to gauge by eye [12]. We believe that this is acceptable for an early prototype system and further experimentation with orientation filters and calibration techniques will improve on this. One limitation of the chosen technology however is the limited frame rate (approximately $50 \mathrm{~Hz}$ ), thus not suitable for very high-speed motion capture.

Our simplified angle of displacement method was sufficient to gauge the approximate accuracy of the system. Future testing of this system will require more detailed analysis using 3D orientation data and time synchronization. Eventually this system will be tested on human subjects to track joint angles. 


\section{ACKNOWLEDGMENTS}

The authors wish to thank the entire Envisage Project team and Bruce Carse for operating the Vicon motion capture system. Thanks also go to Lifelong Health and Wellbeing who are funding this work.

\section{REFERENCES}

[1] E. Roos, "Effectiveness and practice variation of rehabilitation after joint replacement," Curr. Opin. Rheumatol. 2003, vol. 15, pp. 160-162.

[2] J. M. Naylor, R. Mittal, K. Carroll and I. A. Harris, "Introductory insights into patient preferences for outpatient rehabilitation after knee replacement: implications for practice and future research," J. Eval. Clin. Pract. 2011.

[3] S. E. Lamb, S. Robertson and H. Frost, "A randomized controlled trial of exercise to improve mobility and function after elective knee arthroplasty. Feasibility, results and methodological difficulties," Clin. Rehabil. 2002, vol. 16, pp. 200-209.

[4] H. Zhou and H. Hu, "Human motion tracking for rehabilitation-A survey," Biomedical Signal Processing and Control 2007, vol. 3, pp. 1-18.

[5] P. Cappa, H. I. Krebs, L. Masia and N. Hogan, "Design and Characterization of Hand Module for Whole-Arm Rehabilitation Following Stroke," IEEE/ASME Transactions on Mechatronics 2007, vol. 12, pp. 399407.

[6] Duckho Kim, N. Schweighofer, J. Gordon and Younggeun Choi, "An adaptive automated robotic task-practice system for rehabilitation of arm functions after stroke," Robotics, IEEE Transactions on 2009, pp. 556-568.

[7] A. U. Alahakone and S. M. N. A. Senanayake, "A combination of inertial sensors and vibrotactile feedback for balance improvements in therapeutic applications," in Innovative Technologies in Intelligent Systems and Industrial Applications, CITISIA 2009, pp. 5-10.

[8] D.K. Arvind, "Speckled tango dancers: Real-time motion capture of twobody interactions using on-body wireless sensor networks," in Proceedings: Sixth International Workshop on Wearable and Implantable Body Sensor Networks 2009, pp. 312-317.

[9] Xiaoping Yun and E. R. Bachmann, "Design, Implementation, and Experimental Results of a Quaternion-Based Kalman Filter for Human Body Motion Tracking," Robotics, IEEE Transactions on 2006, vol. 22, pp. 12161227.

[10] Z. Huiyu and H. Huosheng, "Upper limb motion estimation from inertial measurements," International Journal of Information Technology 2007, vol. 13.

[11] H. Zhou, H. Hu, T. Stone and N. Harris, "Use of multiple wearable inertial sensors in upper limb motion tracking," Med. Eng. Phys. 2007, vol. 30, pp. 123-133.
[12] V. van Acht, E. Bongers, N. Lambert and R. Verberne, "Miniature wireless inertial sensor for measuring human motions," in Engineering in Medicine and Biology Society EMBS, 29th Annual International Conference of the IEEE, 2007, pp. 6278-6281.

[13] Huiyu Zhou, Huosheng Hu and N. Harris, "Application of wearable inertial sensors in stroke rehabilitation," in Engineering in Medicine and Biology Society EMBS, 27th Annual International Conference of the, 2005, pp. 6825-6828.

[14] Huiyu Zhou and Huosheng Hu, "Inertial motion tracking of human arm movements in stroke rehabilitation," in Mechatronics and Automation, IEEE International Conference, 2005, Vol. 3, pp. 1306-1311.

[15] S. Kobashi, Y. Tsumori, S. Imawaki, S. Yoshiya and Y. Hata, "Wearable knee kinematics monitoring system of MARG sensor and pressure sensor systems," in System of Systems Engineering SoSE, IEEE International Conference on, 2009, pp. 1-6.

[16] M. Newbery, N. Hogan, S. Buerger, M. Sandmann, B. Volpe, D. Lynch, H. Krebs, A. Makiyama and M. Ferraro, "Rehabilitation robotics: pilot trial of a spatial extension for MIT-Manus," Journal of NeuroEngineering and Rehabilitation 2004, vol. 1, pp. 5-5.

[17] G. Rosati, S. Masiero, M. Armani and A. Celia, "Robotic-Assisted Rehabilitation of the Upper Limb After Acute Stroke," Arch. Phys. Med. Rehabil. 2007, vol. 88, pp. 142-149.

[18] L. Dipietro, D. Edwards, N. Hogan, H. I. Krebs, P. T. Huerta, B. T. Volpe, A. Rykman and J. L. Zipse, "Robotic devices as therapeutic and diagnostic tools for stroke recovery," Arch. Neurol. 2009, vol. 66, pp. 1086.

[19] S. K. Charles, J. Celestino, H. I. Krebs, B. T. Volpe, D. Lynch, N. Hogan and D. Williams, "Robot-aided neurorehabilitation: A robot for wrist rehabilitation," in Neural Systems and Rehabilitation Engineering, IEEE Transactions on 2007, pp. 327-335.

[20] G. Kwakkel, H. I. Krebs and B. J. Kollen, "Effects of Robot-Assisted Therapy on Upper Limb Recovery After Stroke: A Systematic Review," Neurorehabil. Neural Repair 2008, vol. 22, pp. 111-121.

[21] P. Giannoni, P. Morasso, M. Casadio and V. Sanguineti, "A proof of concept study for the integration of robot therapy with physiotherapy in the treatment of stroke patients," Clin. Rehabil. 2009, vol. 23, pp. 217-228.

[22] L. D. Paulson and B. A. King, "Motion Capture Moves into New Realms," Computer, vol. 40, pp. 13-16.

[23] S. O. H. Madgwick, R. Vaidyanathan and A. J. L. Harrison, "An Efficient Orientation Filter for IMU and MARG Sensor Arrays," PhD Thesis 2010.

[24] A. Young D, "Wireless Realtime Motion Tracking System using Localised Orientation Estimation," PhD Thesis 2010, pp. 49-56.

[25] SparkFun Electronics, http://www.sparkfun.com/ accessed on 6/23/2010. 